\title{
Calidad de Vida Relacionada con Salud Oral: Impacto de Diversas Situaciones Clínicas Odontológicas y Fac- tores Socio-Demográficos. Revisión de la Literatura
}

\author{
Quality of Life Related to Oral Health: Impact of Various Socio-Demographic \\ Factors and Dental Clinical Situations. Review of Literature
}

\author{
Clarisse Virginia Diaz-Reissner; Irma Casas-García² \& Juan Roldán-Merino³
}

DIAZ-REISSNER, C. V.; CASAS-GARCÍA, I.; ROLDÁN-MERINO, J. Calidad de vida relacionada con salud oral: Impacto de diversas situaciones clínicas odontológicas y factores socio-demográficos. Revisión de la literatura. Int. J. Odontostomat., 11(1):31-39, 2017.

RESUMEN: La calidad de vida relacionada con salud oral es un concepto relativamente nuevo que considera la autopercepción del individuo acerca de su salud, como un componente a ser considerado además del examen clínico odontológico. Se planteó una revisión de la literatura, con el objetivo de buscar evidencia científica sobre el impacto que tienen en la vida diaria diversas patologías orales y diversos factores socio-demográficos, en estudios que utilizaron el cuestionario Perfil de Impacto de Salud Oral, en los últimos 5 años (2009-2013). Se concluye que dicho instrumento es válido para el diagnóstico, plan de tratamiento y pronóstico del paciente, sirviendo como guía en el conocimiento de la percepción del paciente acerca de la afectación en su vida diaria, pudiendo de esta manera prever la adherencia del mismo.

PALABRAS CLAVE: calidad de vida, salud oral, autopercepción, perfil de impacto de enfermedad.

\section{INTRODUCCION}

La calidad de vida, definida por la OMS como "la percepción personal del individuo de su situación de vida, dentro del contexto sociocultural y de valores en que vive, en relación con sus objetivos, expectativas e interés" que a su vez se interrelacionan con diversos factores (de la Fuente-Hernández et al., 2010). Por tanto, abarca un amplio concepto, pues considera que la salud oral se ve afectada por la interacción de la condición de salud oral, social, factores ambientales y salud general, reflejando la satisfacción del individuo con su salud oral (Sischo \& Broder, 2011; Gabardo et al., 2013). Con lo expuesto, se puede considerar que la percepción de salud es una variable multidimensional dependiente tanto de factores internos como externos del individuo (Cohen-Carneiro et al., 2011).
La autoestima y el bienestar, se pueden ver disminuidos por trastornos en la cavidad oral, esta afirmación dio lugar al concepto de Calidad de Vida Relacionada con Salud Oral (CVRSO), lo que podría convertirse en una herramienta que permita comprender el comportamiento del paciente desde una perspectiva más amplia dentro de la práctica clínica, investigación odontológica y prevención de salud oral, así como también en la comunidad, pues estos deberían ser la base para el desarrollo de los programas de salud oral (Bennadi \& Reddy, 2013).

La percepción del paciente resulta importante cuando se evalúa la necesidad de tratamiento, planificación y la espera de un resultado clínico, en el consultorio odontológico. Actualmente, existe un creciente

${ }^{1}$ Odontóloga. Doctoranda en Metodología de la Investigación en Ciencias Biomédicas y Salud Pública. Universitat Autònoma de Barcelona, España.

2 Profesora de la Universitat Autònoma de Barcelona. Departamento de Pediatría, Obstetricia y Ginecología y Medicina Preventiva. España.

3 Profesor Titular del Campus Docent Sant Joan de Déu Fundació Privada. Profesor asociado de la Universitat Autònoma de Barcelona y de la Universitat Rovira i Virgili, España. 
DIAZ-REISSNER, C. V.; CASAS-GARCÍA, I.; ROLDÁN-MERINO, J. Calidad de vida relacionada con salud oral: Impacto de diversas situaciones clínicas odontológicas y factores socio-demográficos. Revisión de la literatura. Int. J. Odontostomat., 11(1):31-39, 2017.

reconocimiento de que la salud oral tiene un impacto en la vida social y psicológica del individuo. Por tanto, es importante determinar las causas por las cuales los pacientes tienden a buscar tratamiento y en qué medida esto afecta su calidad de vida (Caglayan et al., 2009).

Su importancia radica en que permite un cambio en el criterio odontológico hacia la definición de metas y resultados de tratamientos basados en la experiencia emocional y social, y la funcionalidad; el paciente pasa a tener un papel más activo en el tratamiento. Además, resulta útil para medir el impacto de la inequidad en salud oral; que ya ha sido reconocido por la OMS en Programas de Salud Oral Global (Sischo \& Broder).

Por esta razón, se planteó una revisión narrativa con el objetivo de buscar evidencia científica acerca de factores socio-demográficos y patologías orales que generan repercusiones en la calidad de vida oral. Se discuten las diversas aplicaciones clínicas y se presenta el modo como estas influyeron complementando al diagnóstico clínico, describiendo las ventajas de su utilización en el proceso de diagnóstico, tratamiento y control del paciente.

Los artículos incluidos en este trabajo fueron publicados en PubMed (Biblioteca Nacional de Medicina de los Estados Unidos de los Institutos Nacionales de Salud) y Redalyc (Red de Revistas Científicas de América Latina y el Caribe, España y Portugal) en los últimos cinco años; los cuales utilizaron el cuestionario Perfil de Impacto de Salud Oral (OHIP por sus siglas en inglés), exponiendo su uso en diversas situaciones clínicas odontológicas y su impacto en la calidad de vida oral de los pacientes, en distintos grupos etarios y poblaciones. Así como también, artículos de revisión de la literatura y revisión sistemática sobre calidad de vida relacionada con salud oral.

Calidad De Vida Relacionada Con Salud ORAL (CVRSO) Instrumentos de evaluación de la autopercepción. Medir la calidad de vida conforme el estado bucodental resulta subjetivo al estar influenciado por distintos factores, que no pueden ser observados de manera directa, motivo por el cual se han desarrollado instrumentos de medición representadas por dimensiones que pretenden valorar la percepción psicosocial personal de cada individuo, utilizadas para estimar y complementar la evaluación física (de la Fuente-Hernández et al.)
El Perfil de Impacto de Salud Oral conocido por sus siglas en inglés OHIP (Oral Health Índex Profile) es uno de los instrumentos más utilizados para la evaluación de la percepción sobre salud oral en pacientes y su impacto en la calidad de vida diaria (LópezJornet et al., 2009; Montero-Martín et al., 2009). Si bien, existen otros instrumentos de medición de la calidad de vida oral, el OHIP es el que ha sido más estudiado (Sanders et al., 2009a), cuyo enfoque está basado en la frecuencia de los impactos percibidos (Montero et al., 2010).

La versión extendida original (OHIP-49) consta de 49 ítems que fueron desarrollados sobre la base de un modelo teórico de la OMS, que posteriormente fue adaptado por Locker (Montero-Martín et al.), desarrollada en Australia (Pereira et al., 2009). La versión original fue simplificada a una versión de 14 ítems (OHIP-14), que ha demostrado ser un cuestionario confiable, a pesar de ser corto (Montero-Martín et al.). Demostrando tener un constructo coherente con el marco teórico, que presenta una clara discriminación de sus componentes (Montero et al.).

El cuestionario cuenta con siete dominios que son: limitación funcional, dolor físico, malestar psicológico, discapacidad física, discapacidad psicológica, discapacidad social, y minusvalía. Además, se ha demostrado que es confiable, sensible a los cambios y exhibe una adecuada consistencia interna transcultural (Khalifa et al. 2013; López-Jornet et al.; Montero-Martín et al.). Ya ha sido validado en más de 20 idiomas (Sanders et al., 2009b), tales como: chino, francés, alemán, japonés, malayo, portugués, inglés, somalí, sueco, etc. (Montero-Martín et al.)

El OHIP-14SP, que corresponde a la versión en español, ha demostrado ser un instrumento preciso, válido y confiable para evaluar la salud bucodental de la calidad de vida entre los adultos de la población española, chilena (Montero-Martín et al.) y mexicana (Castrejón-Pérez et al., 2010), validados recientemente. Por tanto, es ampliamente utilizado en diversidad de estudios, tanto transversales como longitudinales (Sousa et al., 2009) y aceptado internacionalmente (Montero et al.). Se presenta a continuación (Tabla I) la versión en español del cuestionario. La misma tienen categorías: nunca (0), casi nunca (1), ocasionalmente (2), frecuentemente (3) y muy frecuentemente (4). Para obtener el puntaje se suman los valores, pudiendo por tanto tener un mínimo de 0 puntos y máximo de 56 puntos por cada paciente. Entonces, puntajes bajos indican mejor calidad de vida auto percibida y 


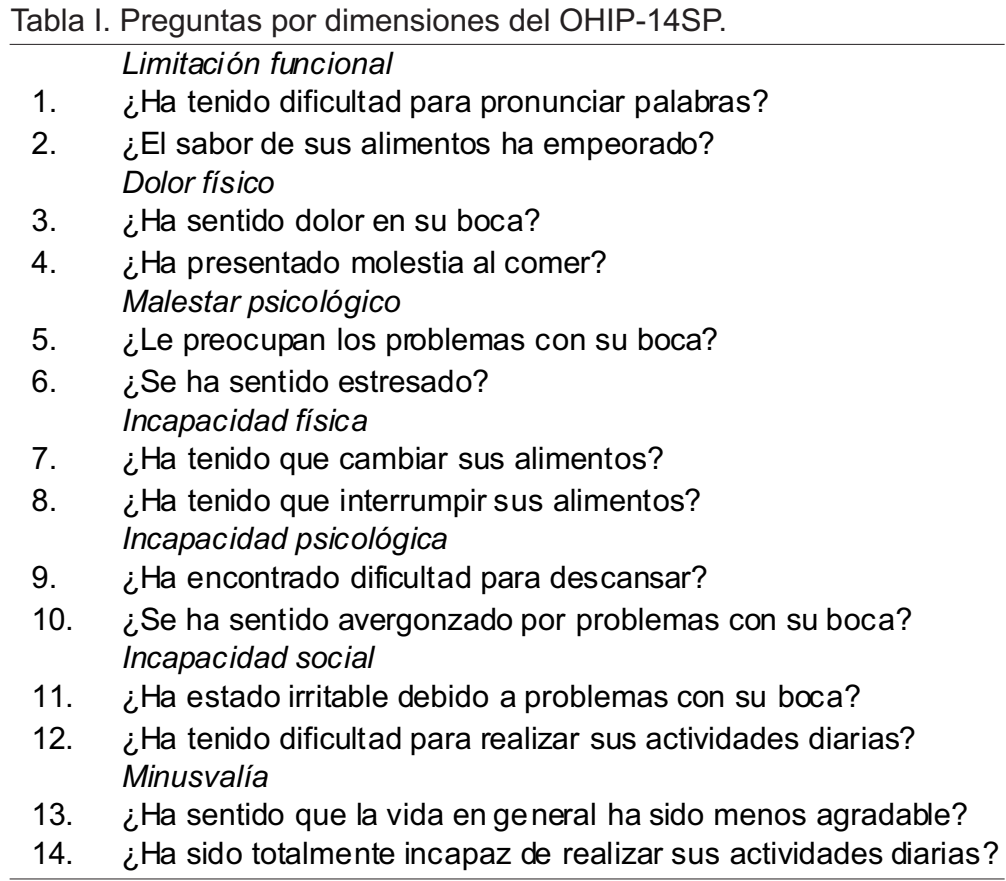

puntajes altos indican peor autopercepción de la calidad de vida (de la Fuente-Hernández et al.) También, se puede expresar como autopercepción negativa y positiva.

En cuanto a la forma de administración, según sea cuestionario o entrevista, se ha encontrado que la misma no influye en las puntuaciones totales del OHIP; pero la entrevista presentó una significativamente mayor tasa de respuesta (Sousa et al.).

Factores clínicos, socio-demográficos y psicológicos. La autopercepción de la calidad de vida relacionada con salud oral puede verse afectada por diversos factores, tanto sociodemográficos como clínicos. En un estudio realizado en pacientes estadounidenses con una edad promedio de 40 años, encontraron que las mujeres tenían más probabilidad de tener autopercepción negativa, así como también, ser afroamericano constituyó un factor de riesgo; mientras que al analizar todas las variables consideradas juntas para predecir la autopercepción resultaron también predictores la edad, la procedencia, el tipo de odontólogo según sea generalista o especialista y los años de experiencia del odontólogo (Botello-Harbaum et al., 2012).

En otro estudio, con pacientes estadounidenses, se encontró que los niveles de autopercepción se incrementaban con mayores niveles de pérdida den- tal, necesidad de tratamiento percibida, falta de seguro dental privado y bajos ingresos económicos, similar a lo ocurrido en la población australiana (Sanders et al., 2009a), donde además se encontró que la puntuación media del OHIP se reducía en 1,7 unidades con cada aumento del cuartil de ingreso de los hogares, siendo esto más pronunciado en otros países (Sanders et al., 2009b).

No obstante, la mitad de los pacientes adultos que acudieron al Departamento de Diagnóstico y Radiología de la Facultad de Odontología de la Universidad de Ataturk, Turquía lo hicieron por dolor y caries dental. Encontrándose que las mujeres tienden significativamente a tener una autopercepción negativa acerca de su calidad de vida oral sobre con respecto a los hombres. Así también obtuvieron el puntaje más alto, con percepción significativamente negativa, los pacientes que no recibieron educación escolar o estudiaron solo la primaria, y los que tenían el hábito de fumar (Caglayan et al.). En adultos mayores mexicanos se encontró que tuvo mayor impacto en el sexo femenino, viéndose afectadas las dimensiones malestar psicológico, dolor físico y funcional (de la FuenteHernández et al.)

Por otro lado, encontraron que la presencia de dientes cariados, con necesidad de extracción o tratamiento de endodoncia fueron los principales factores que afectaron la calidad de vida oral en adultos espa- 
ñoles, debido a que estas condiciones están generalmente asociadas con la presencia de dolor. Así también, los dientes cariados en zonas visibles, como ser premolares, caninos o incisivos demostraron ser de suma importancia debido a su impacto en la afectación de las dimensiones social y psicológica del individuo (Montero-Martín et al.). En adultos griegos se encontraron puntuaciones altas para las dimensiones de limitación funcional, dolor físico, discapacidad y malestar psicológico, resultando el nivel educativo la única variable significativa (Papaioannou et al., 2011).

Según un estudio de realizado en Brasil, las puntuaciones medias del OHIP tenían tendencia a disminuir conforme aumentaba la edad, variando muy poco la puntuación para las variables sociodemográficas y estado de salud oral (Cohen-Carneiro et al., 2010). Mientras que en otro estudio realizado en adultos mayores brasileros se asociaron con un impacto negativo de salud oral el sexo femenino, el nivel socioeconómico bajo, tener tres pares dentarios en oclusión en sector posterior, una o más lesiones de caries sin tratar, haber acudido a la última cita del odontólogo para tratamientos restauradores y no preventivos, una escasa percepción acerca de la importancia de los cuidados dentales y una autovaloración de salud oral mala; siendo este último el más elevado, aumentando en tres veces la probabilidad de riesgo (Ulinski et al., 2013). Por otra parte, en otro estudio concluyeron que las variables sociodemográficas y condiciones orales explican un pequeño porcentaje de la variación de la calidad de vida, siendo los más afectados las dimensiones físicas y psicológicas, disminuyendo la calidad de vida conforme aumenta la edad (Bianco et al., 2010). En India, la perdida dentaria se asoció con la edad, teniendo un efecto independiente en la CVRSO (Jain et al., 2012). En adultos y adultos mayores canadienses siendo los impactos más frecuentes el dolor físico y malestar psicológico, siendo más propensos a un impacto negativo los que residen en áreas rurales y tienen educación secundaria o inferior (Kotzer et al., 2012).

Asimismo, al comparar la autopercepción de los pacientes sobre su salud oral entre pacientes dentados y edéntulos, se observó que el promedio del puntaje fue más alto para los pacientes edéntulos que utilizaban prótesis dental, recibían atención dental en servicios públicos, asistían irregularmente a las consultas y tenían ingresos económicos más bajos. Siendo los puntajes más altos para eldolor percibido en la cavidad oral, la incomodidad para masticar alimentos y el malestar psicológico debido al estado de salud oral (Locker
\& Quiñonez, 2009). La pérdida de dientes se asoció con la calidad relacionada con la salud de vida oral negativa. Además el número, la ubicación y la distribución de los dientes que faltan también afectan la percepción (Zhang et al., 2013), así como la pérdida dentaria total tuvo un fuerte impacto en la calidad de vida (Silva et al., 2010a).

También, otro factor que parece incidir es la emocionalidad negativa, individuos con puntajes más altos tenían un mayor riesgo de haber perdido al menos un diente debido a caries y tenían un mayor riesgo de tener tres o más superficies cariadas (Thomson et al., 2011). Como resultado de un estudio de cohorte desde el nacimiento hasta la edad adulta en Nueva Zelanda encontraron que también la autopercepción de la madre cuando el niño es pequeño incide tres décadas después en el dominio psicológico (Shearer et al., 2011).

Por último, en un estudio realizado en mujeres embarazadas brasileñas, el OHIP fue bajo, constituyendo factores de riesgo haber realizado la última visita al odontólogo hace más de 8 años y tener un índice CPOD mayor a 13. Las dimensiones más afectadas fueron la dimensión disconformidad psicológica, dolor físico e incapacidad psicológica (Rosell et al., 2013). En otro estudio en embarazadas se comparó la percepción de CVRSO en chilenas e inmigrantes peruanas, no encontrándose diferencia significativa, pero el $33 \%$ consideró su salud oral como mala (Misrachi et al., 2009).

Alteraciones en tejidos blandos. En cuanto a la alteración de los tejidos blandos, fueron evaluados los factores que influían en la calidad de vida en pacientes con aparatología ortodóncica fija y agrandamiento gingival, encontrándose que los factores de riesgo para una percepción negativa estaban dados por el aumento del nivel de agrandamiento gingival, raza no blanca, bajos ingresos, personas con sobrepeso, con maloclusión severa o muy severa (Zanatta et al., 2012). En un estudio realizado en el Departamento de Enfermedades de la Mucosa Oral, Hospital de Shanghai, se comparó la puntuación media del OHIP entre pacientes con enfermedad de la mucosa oral y pacientes sanos, siendo el promedio significativamente mayor en los pacientes enfermos (Liu et al., 2012). En otro estudio, la estomatodinia fue la patología que reportó peor calidad de vida, en el dominio de función física (López-Jornet et al.).

Sin embargo, la presencia de enfermedad periodontal no se correlacionó con una disminución 
del impacto en la calidad de vida oral, a menos que la enfermedad esté muy avanzada y acompañada de movilidad dental (Montero-Martín et al.). Aunque, otro estudio arrojó como resultado que la periodontitis crónica severa, si presentaba una asociación significativa, siendo los más afectados los dominios dolor físico y discapacidad física (Al Habashneh et al., 2012).

Cirugía oral. Con respecto a cirugía oral, en pacientes sometidos a extracción de terceros molares mandibulares se observó un deterioro significativo en la CVRSO en el postoperatorio inmediato, en los cinco días posteriores a la extracción dental (Deepti et al., 2009). Mientras que en otro estudio, al realizar un análisis de regresión múltiple del efecto de las variables independientes (edad y sexo de los sujetos, las complicaciones intraoperatorias, la duración de la extracción, y complicaciones de la cicatrización con osteitis alveolar) en la calidad de vida (variable dependiente) en pacientes con extracción dental no se encontró asociación significativa entre estas variables y el deterioro en la calidad de vida (Adeyemo et al., 2012).

Por otra parte, en otro estudio donde compararon la percepción de la calidad de vida antes de la extracción del tercer molar, con cada día postoperatorio durante una semana y al mes, encontrándose diferencia significativa en todos los 7 días postoperatorios, restableciéndose al mes. Las variables clínicas que influyeron fueron la presencia de dolor postoperatorio, que tuvieron una puntuación significativamente mayor que aquellos pacientes que no tuvieron quejas; también que el diente se haya encontrado impactado, parcialmente o totalmente recubierto por mucosa, dado que en estos últimos el tiempo de cirugía fue mayor en promedio, por lo que la puntuación media, si bien inicialmente fue mayor, en los primeros se mantuvo, mientas que en el postoperatorio fue mayor para los últimos. Así también, el nivel de retención III de la Clasificación Pell y Gregory del tercer molar, mostró el mayor incremento en la puntuación media del OHIP (Kieffer et al., 2012).

Ahora bien, pacientes adultos con recesiones gingivales clases I y II de Miller que se sometieron a un tratamiento quirúrgico, arrojaron resultados de una reducción del OHIP a los 3 meses, encontrándose satisfechos con el aspecto final del proceso (Hansmeier \& Eickholz, 2010).

Mientas que, en pacientes diagnosticados de deformidad esquelética de ortodoncia, que se les realizó osteotomía maxilar anterior junto con la correc- ción de ortodoncia, fueron evaluados al inicio del tratamiento pre-quirúrgico, 8 semanas después de la operación, y 24 semanas después de la operación; el resultado mostró una leve mejoría en la CVRSO inmediatamente después de la cirugía, mientras que se observó la calidad y la percepción de la mejora estética del paciente sólo a las 24 semanas después de la osteotomía (Kavin et al., 2012).

Disfunciones del sistema estomatognático. En relación a disfunciones del sistema estomatognático, al evaluar la función masticatoria desde la autopercepción del paciente, con 20 alimentos de consumo común en japoneses, se encontró que cuando mejora la capacidad masticatoria, el deterioro de la calidad de vida disminuye (Baba et al., 2009). Mientras que en otro estudio, el aumento de la capacidad de masticar se correlacionó con las puntuaciones más bajas OHIP y estuvo influenciado por el sexo, años de escolaridad, demanda de tratamiento y estado de los dientes (Inukai et al., 2010). En una población adulta brasileña se encontró que a medida que aumenta la edad ( $\geq 40$ años) disminuye el número de dientes y la capacidad de masticar, generando un impacto negativo en la calidad de vida, afectando 5 de los 7 dominios: limitación funcional, dolor físico, bienestar psicológico, discapacidad física o psicológica (Bortoluzzi et al., 2012). Así también, en otra población brasileña, a la misma edad y con necesidad de prótesis parcial y total declarada, se encontró un disminución en la calidad de vida (Miotto et al., 2012).

Por otro lado, al evaluar la calidad de vida oral en mujeres con trastornos de la Articulación TemporoMandibular (ATM) se encontró que la calidad de vida oral se ve afectada por dicha patología, pues a mayor severidad del trastorno la autopercepción de los problemas orales es mayor (Pereira et al.).

Maloclusiones. Se han considerado pacientes con maloclusiones, de los que asistieron al consultorio de ortodoncia con y sin tratamiento, en el grupo de tratamiento fue afectado significativamente el dominio limitación física (Navabi et al., 2012). En otro estudio, el dominio malestar fue el que registró mayor impacto, así como también estuvo afectado en el grupo 15-18 años de edad que mostró el mayor impacto en su calidad de vida debido a la maloclusión. Los participantes con una formación universitaria reportaron un impacto significativamente mayor en comparación con los participantes con una educación secundaria (Masood et al., 2013). 
Por otra parte, de los pacientes con maloclusión severa, las mujeres reportaron la media de puntuación OHIP mayor, niveles más altos de dolor, y tenían más trastornos temporo-mandibulares severos que los hombres, pero la diferencia no fue estadísticamente significativa. Entre los hombres, las características oclusales fueron directamente relacionados con la calidad de la salud oral (Rusanen et al., 2012). Se evaluó como incide en la calidad de vida oral la presencia de maloclusiones severas y deformidades faciales, antes del tratamiento dental, y la influencia del género en dicha percepción. Comparados con pacientes con normo oclusión, los pacientes con maloclusión severa presentaron alto impacto en la calidad de vida oral, siendo más frecuente en mujeres, encontrándose que, han sido más afectados por problemas con sus dientes o prótesis aquellos pacientes con mordida cruzada lateral en comparación con aquellos pacientes con dimensiones normales transversales de los dientes laterales; los pacientes con mordida abierta reportaron más molestias al comer que los pacientes con superposición vertical normal o mordida profunda; mientras que los pacientes con resalte negativo se mostraron más irritables que los de resalte positivo. Los pacientes con maloclusión Clase III reportaron una dieta más satisfactoria y fueron más sensibles en relación con sus dientes o prótesis que los pacientes de Clase II (Rusanen et al., 2010).

Patologías que afectan la cavidad oral. Atendiendo a patologías que afectan la cavidad oral, se encontró que la calidad de vida oral se redujo significativamente a medida que aumentaba la gravedad de la Osteonecrosis Mandibular (OM) asociada a bifosfonatos (Miksad et al., 2011).

Al mismo tiempo, en pacientes con dolor temporo-mandibular crónico, la media de puntuación OHIP para los grupos con y sin tinitus no presentaron diferencias estadísticamente significativas para la intensidad del dolor, duración del dolor y las puntuaciones OHIP (Calderon et al., 2012).

Prótesis dental. En pacientes adultos con al menos una prótesis completa que acudieron al Departamento de Prótesis Bucofacial de la Universidad Complutense (Madrid), se investigaron las diferencias de impacto en la salud oral de la calidad de vida en pacientes portadores de prótesis total, conforme a características socio-demográficas, factores relacionados con la prótesis y estado oral. Los dominios más afectados fueron "limitación funcional" y "dolor físico", seguido de "discapacidad física". Los pacientes sin estomatitis protésica y los que necesitan reparar o cambiar sus prótesis, registraron significativamente más altos puntajes totales OHIP. El mayor impacto percibido fue encontrado en las personas mayores de 40 años $(O R=2,37)$, con una necesidad declarada de prótesis parciales $(\mathrm{OR}=2,77)$, y la prótesis removible $(\mathrm{OR}=2,29)$ (Miotto et al.).

Como resultado de un estudio realizado en Bosnia, al comparar la calidad de vida en pacientes con prótesis dental desgastada y con prótesis dental nueva, se encontró que estos últimos presentaron una mejor calidad de vida, mejorando significativamente su calidad de vida a la primera y segunda semana de instalación de la prótesis. Aquellos con necesidad de prótesis, que nunca tuvieron una, reportaron significativamente peor calidad de vida que los que la cambiaron (Hadzipasic-Nazdrajic, 2011). Así también, en un estudio en brasileños el uso de prótesis inadecuadas generó impactos negativos en la calidad de vida (Silva et al, 2010b).

Por su parte, pacientes adultos españoles portadores de prótesis convencional registraron puntajes significativamente más altos de OHIP, cuando no presentaban estomatitis y necesitaban cambiar o repararla. Siendo los dominios más afectados la limitación funcional, dolor físico y discapacidad física (Perea et al., 2013).

Finalmente, al comparar la CVRSO en pacientes edéntulos rehabilitados con prótesis dentosopotadas sobre implantes y prótesis fija, no se encontró diferencia significativa entre ambas (Zani et al., 2009).

\section{DISCUSION}

El OHIP constituye una ayuda importante en la comprensión de las necesidades de salud oral y en el desarrollo de estrategias para el control y la reducción de las patologías orales, así como también para la promoción de la salud oral (Gabardo et al.).

Para hacer que estos hallazgos sean clínicamente relevantes, así como también para la interpretación de los estudios transversales y longitudinales, primeramente se podría aplicar el criterio de Reissmann, tanto para pacientes individuales como grupos de población (Reissmann et al., 2013). Si bien, se ha demostrado su utilidad en diversas po- 
blaciones (Sischo \& Broder), tienen como limitación fundamental que la salud de la población es una medida del contexto, por tanto, no abarca solamente la salud oral (Slade, 2012).

En otro estudio, encontraron que un impacto negativo cada día en salud oral en el último mes llevaría a una autopercepción negativa con el OHIP-49, lo que impulsaría al paciente a realizar la consulta odontológica. Es decir que 15,2 es la frecuencia por cada punto del OHIP, con la que al multiplicarla por el puntaje total del OHIP se obtendría el valor de impactos previos a la búsqueda de tratamiento (Reissmann et al.).

El análisis de la calidad de vida referente a las condiciones de salud oral podrían tener efecto sustancial en la toma de decisiones y la adherencia al tratamiento dental (Botello-Harbaum et al.), siendo más efectiva su aplicación a manera de entrevista (Sousa et al.). Se ha encontrado que en adultos influyeron negativamente en la CVRSO factores clínicos, sociodemográficos y subjetivos (Ulinski et al.).

Por lo expuesto, estos indicadores subjetivos deberían utilizarse como complemento al diagnóstico clínico, de manera a determinar el tratamiento, la posibilidad de mejora y CVRSO en el paciente (LópezJornet et al.; Miotto et al.).

Se debe considerar también, que otro factor de riesgo para la caries dental es la personalidad. Aquellos con emociones negativas reportaron peor salud oral, encontrándose en ellos un mayor número de caries y dientes perdidos. Las actitudes y valores pueden ser alterados mediante intervenciones breves, que podrían ser útiles para crear cambio de conducta en odontología preventiva (Thomson et al.). También influye en la adultez la autopercepción de la madre (Shearer et al.), recibir atención odontológica en servicios públicos, visitar irregularmente al odontólogo, bajos ingresos económicos (Locker \& Quiñonez; Sanders et al., 2009a), el nivel de educación (Caglayan et al.; Inukai et al.; Kotzer et al.; Papaioannou et al.) y que resida en el área rural (Kotzer et al.).

Varios estudios coincidieron que las mujeres tienden a tener una mayor percepción negativa de su salud oral que los hombres (Caglayan et al.; de la Fuente-Hernández et al.; Inukai et al.; Rusanen et al., 2010; Ulinski et al.) y también a medida que aumenta la edad lo hace la percepción negativa (Bianco et al.; CohenCarneiro et al., 2010; Jain et al.).
En pacientes adultos se encontró que una calidad de vida positiva está relacionada con la presencia de por lo menos 10 dientes en cada arcada, prefiriendo los dientes naturales (Baba et al.; Bortoluzzi et al.; Jain et al.; Sanders et al., 2009b; Zhang et al.), además agrega que su disminución afectan la capacidad de masticar (Inukai et al.), tendiendo un fuerte impacto negativo la pérdida total de dientes (Silva et al., 2010a). También en pacientes en los que se le realizó osteotomía de la mandíbula, debido a una mejora a los 2 meses de la estética, función, salud oral y satisfacción (Kavin et al.), y en pacientes que recibieron una prótesis removible nueva (Hadzipasic-Nazdrajic).

Sin embargo, los trastornos de la ATM no alteraron la calidad de vida (Calderon et al.), resultado distinto al encontrado en otro estudio donde que vio en mujeres con niveles más elevados de dolor y patología más severa, aunque la oclusión se encontró afectada en los hombres (Rusanen et al., 2012). Sin embargo, en otro estudio encontraron asociación entre la gravedad del trastorno y la calidad de vida (Pereira et al.).

La presencia de maloclusión tuvo un impacto negativo en la calidad de vida de adultos jóvenes, especialmente en el dominio psicológico (Masood et al.) en coincidencia con otro estudio, aunque estos fueron mayores en los hombres (Rusanen et al., 2010). En adultos con ortodoncia, el agrandamiento gingival influyó negativamente en su calidad de vida (Zanatta et al.). Al utilizar tratamiento ortodóncico los pacientes mejoraron la dimensión física, con respecto a aquellos que necesitan pero nunca se realizaron el tratamiento (Navabi et al.).

Según Perea et al., el uso de prótesis total mucosoportada en adultos, tiene impactos negativos en la calidad de vida, afectando más la necesidad de reparación o sustitución de las inferiores que tienen como antagonista una prótesis removible, similar a lo reportado en otro estudio (Silva et al., 2010b). Sin embargo, la estomatitis subprotésica estaba asociada a otra enfermedad, pudiendo influir en una percepción negativa de malestar con la prótesis (Perea et al.). No existiendo diferencia en la percepción con respecto al tipo de prótesis, ya sea esta implanto-soportada o fija (Zani et al.).

La CVRSO se vio deteriorada en pacientes con enfermedad en la mucosa oral (Liu et al.), en el primer mes de una extracción quirúrgica de terceros molares (Adeyemo et al.; Kieffer et al.), conforme la gravedad de la enfermedad periodontal (Al Habashneh et al.). También, en pacientes con cáncer OM asociado a 
bifosfonato (Miksad et al.), con el síndrome de la boca ardiente (López-Jornet et al.), con necesidad declarada de prótesis total o parcial (Miotto et al.), con periodontitis muy avanzada (Montero-Martín et al.).

En cirugía, la CVRSO se vio mejorada a los 3 meses de realizar cobertura de raíces (Hansmeier \& Eickholz), a los 6 días de la extracción del tercer mo$\operatorname{lar}$ (Deepti et al.).

\section{CONCLUSIONES}

La evaluación de la calidad de vida relacionada a la salud oral representa un instrumento válido para el diagnóstico, plan de tratamiento y pronóstico del paciente, permitiendo prever el nivel de adherencia del paciente al plan presentado o ajustando el plan a las percepciones del paciente, lo cual en última instancia garantiza el éxito del mismo. La aplicación del mismo lleva minutos y la informaciónproveída es de suma importancia para el buen desarrollo de la relación paciente-profesional.

DIAZ-REISSNER, C. V.; CASAS-GARCÍA, I.; ROLDÁN-MERINO, J. Quality of life related to oral health: Impact of various socio-demographic factors and dental clinical situations. Review of literature. Int. J. Odontostomat., 11(1):31-39, 2017.

ABSTRACT: The quality of life related to oral health is a relatively new concept that considers the self-perception of the individual about their health as a component to be further considered the dental clinical examination. A narrative review was proposed with the aim of seeking scientific evidence of the impact on daily life various oral pathologies and various socio-demographic factors in studies using Impact Profile Questionnaire Oral Health in the last 5 years (2009-2013). We conclude that the instrument is valid for the diagnosis, treatment plan and prognosis of the patient, serving as a guide in understanding the patient's perception about the effect on their daily lives, and can thus provide the same grip.

KEY WORDS: quality of life, oral health, selfconcept, sickness impact profile.

\section{REFERENCIAS}

Adeyemo, W. L.; Taiwo, O. A.; Oderinu, O. H.; Adeyemi, M. F.; Ladeinde, A. L. \& Ogunlewe, M. O. Oral health-related quality of life following non-surgical (routine) tooth extraction: A pilot study. Contemp. Clin. Dent., 3(4):427-32, 2012.

Al Habashneh, R.; Khader, Y. S. \& Salameh, S. Use of the Arabic version of Oral Health Impact Profile-14 to evaluate the impact of periodontal disease on oral health-related quality of life among Jordanian adults. J. Oral Sci., 54(1):113-20, 2012.

Baba, K.; John, M. T.; Inukai, M.; Aridome, K. \& Igarahsi, Y. Validating an alternate version of the chewing function questionnaire in partially dentate patients. B. M. C. Oral Health, 9:9, 2009.

Bennadi, D. \& Reddy, C. V. K. Oral health related quality of life. J. Int. Soc. Prev. Community Dent., 3(1):1-6, 2013.

Bianco, V. C.; Lopes, E. S.; Borgato, M. H.; Moura e Silva, P. \& Marta, $\mathrm{S}$. N. The impact on life quality due to oral conditions in people fifty years or above. Cien. Saude Colet., 15(4):2165-72, 2010.

Bortoluzzi, M. C.; Traebert, J.; Lasta, R.; Da Rosa, T. N.; Capella, D. L. \& Presta, A. A. Tooth loss, chewing ability and quality of life. Contemp. Clin. Dent., 3(4):393-7, 2012.

Botello-Harbaum, M. T.; Matthews, A. G.; Collie, D.; Vena, D. A.; Craig, R. G.; Curro, F. A.; Thompson, V. P.; Broder, H. L. \& PEARL Network. Level of oral health impacts among patients participating in PEARL: a dental practice-based research network. Community Dent. Oral Epidemiol., 40(4):332-42, 2012.

Caglayan, F.; Altun, O.; Miloglu, O.; Kaya, M. D. \& Yilmaz, A. B. Correlation between oral health-related quality of life (OHQoL) and oral disorders in a Turkish patient population. Med. Oral Patol. Oral Cir. Bucal, 14(11):e573-8, 2009.

Calderon, P. dos S.; Hilgenberg, P. B.; Rossetti, L. M. N.; Laurenti, J. V. E. H. \& Conti, P. C. R. Influence of tinnitus on pain severity and quality of life in patients with temporomandibular disorders. J. Appl. Oral Sci., 20(2):170-3, 2012.

Castrejón-Pérez, R. C.; Borges-Yáñez, S. A. \& Irigoyen-Camacho, M. E. Validation of an instrument for measuring the effects of oral health on the quality of life of older adults in Mexico. Rev. Panam. Salud Publica, 27(5):321-9, 2010.

Cohen-Carneiro, F.; Rebelo, M. A.; Souza-Santos, R.; Ambrosano, G. M.; Salino, A. V. \& Pontes, D. G. Psychometric properties of the OHIP-14 and prevalence and severity of oral health impacts in a rural riverine population in Amazonas State, Brazil. Cad. Saude Publica, 26(6):1122-30, 2010.

Cohen-Carneiro, F.; Souza-Santos, R. \& Rebelo, M. A. B. Quality of life related to oral health: contribution from social factors. Ciênc. Saúde Coletiva, 16 Suppl. 1:1007-15, 2011.

Deepti, C.; Rehan, H. S. \& Mehra, P. Changes in quality of life after surgical removal of impacted mandibular third molar teeth. J. Maxillofac. Oral Surg., 8(3):257-60, 2009.

de la Fuente-Hernández, J.; Sumano-Moreno, O.; SifuentesValenzuela, M. C. \& Zelocuatecatl-Aguilar, A. Impacto de la salud bucal en la calidad de vida de adultos mayores demandantes de atención dental. Univ. Odontol., 29(63):83-92, 2010.

Silva, M. E. de S.; Villaça, Ê. L.; de Magalhães, C. S. \& Ferreira, E. F. Impacto da perda dentária na qualidade de vida. Ciênc. Saúde Coletiva, 15(3):841-50, 2010a.

Silva, M. E. de S.; de Magalhães, C. S. \& Ferreira, E. F. Perda dentária e expectativa da reposição protética: estudo qualitativo. Ciênc. Saúde Coletiva, 15(3):813-20, 2010b.

Gabardo, M. C. L.; Moysés, S. T. \& Moysés, S. J. Self-Rating of oral health according to the oral health impact profile and associated factors: a systematic review. Rev. Panam. Salud. Publica, 33(6):43945, 2013.

Hadzipasic-Nazdrajic, A. Quality of life with removable dentures. Mater. Sociomed., 23(4):214-20, 2011

Hansmeier, U. \& Eickholz, P. Effect of root coverage on oral health impact profile (G49): A pilot study. Int. J. Dent., 2010:252303, 2010.

Inukai, M.; John, M. T.; Igarashi, Y. \& Baba, K. Association between perceived chewing ability and oral health-related quality of life in partially dentate patients. Health Qual. Life Outcomes, 8:118, 2010.

Jain, M.; Kaira, L. S.; Sikka, G.; Singh, S.; Gupta, A.; Sharma, R.; Sawla, L. \& Mathur, A. How do age and tooth loss affect oral health impacts and quality of life? A study comparing two state samples of gujarat and rajasthan. J. Dent. (Tehran), 9(2):135-44, 2012. 
Kavin, T.; Jagadesan, A. G. P. \& Venkataraman, S. S. Changes in quality of life and impact on patients perception of esthetics after orthognathic surgery. J. Pharm. Bioallied Sci., 4(Suppl. 2):S290-3, 2012.

Khalifa, N. F.; Allen, P. F.; Abu-bakr, N. H. \& Abdel-Rahman, M. E. Psychometric properties and performance of the Oral Health Impact Profile (OHIP-14s-ar) among Sudanese adults. J. Oral Sci., 55(2):123-32, 2013.

Kieffer, J. M.; van Wijk, A. J.; Ho, J. P. \& Lindeboom, J. A. H. The internal responsiveness of the Oral Health Impact Profile-14 to detect differences in clinical parameters related to surgical third molar removal. Qual. Life Res., 21(7):1241-7, 2012.

Kotzer, R. D.; Lawrence, H. P.; Clovis, J. B. \& Matthews, D. C. Oral health-related quality of life in an aging Canadian population. Health Qual. Life Outcomes, 10:50, 2012.

Liu, L. J.; Xiao, W.; He, Q. B. \& Jiang, W. W. Generic and oral quality of life is affected by oral mucosal diseases. B. M. C. Oral Health, 12:2, 2012.

Locker, D. \& Quiñonez, C. Functional and psychosocial impacts of oral disorders in Canadian adults: a national population survey. J. Can. Dent. Assoc., 75(7):521, 2009.

López-Jornet, P.; Camacho-Alonso, F. \& Lucero-Berdugo, M. Measuring the impact of oral mucosa disease on quality of life. Eur. J. Dermatol., 19(6):603-6, 2009.

Masood, Y.; Masood, M.; Zainul, N. N.; Araby, N. B.; Hussain, S. F. \& Newton, T. Impact of malocclusion on oral health related quality of life in young people. Health Qual. Life Outcomes, 11:25, 2013.

Miksad, R. A.; Lai, K. C.; Dodson, T. B.; Woo, S. B.; Treister, N. S.; Akinyemi, O.; Bihrle, M.; Maytal, G.; August, M.; Gazelle, G. S. \& Swan, J. S. Quality of life implications of bisphosphonate-associated osteonecrosis of the jaw. Oncologist, 16(1):121-32, 2011.

Miotto, M. H.; Barcellos, L. A. \& Velten, D. B. Avaliação do impacto na qualidade de vida causado por problemas bucais na população adulta e idosa em município da Região Sudeste. Cien. Saude Colet., 17(2):397-406, 2012.

Misrachi, C.; Ríos, M.; Morales, I.; Urzúa, J. P. \& Barahona, P. Calidad de vida y condicion de salud oral en embarazadas chilenas e inmigrantes peruanas. Rev. Peru. Med. Exp. Salud Pública, 26(4):455-61, 2009.

Montero, J.; Bravo, M.; Vicente, M. P.; Galindo, M. P.; López, J. F. \& Albaladejo, A. Dimensional structure of the oral health-related quality of life in healthy Spanish workers. Health Qual. Life Outcomes, 8:24, 2010.

Montero-Martín, J.; Bravo-Pérez, M.; Albaladejo-Martínez, A.; Hernández-Martín, L. A. \& Rosel-Gallardo, E. M. Validation the Oral Health Impact Profile (OHIP-14sp) for adults in Spain. Med. Oral Patol. Oral Cir. Bucal, 14(1):E44-50, 2009.

Navabi, N.; Farnudi, H.; Rafiei, H. \& Arashlow, M. T. Orthodontic treatment and the oral health-related quality of life of patients. $J$. Dent. (Tehran), 9(3):247-54, 2012.

Papaioannou, W.; Oulis, C. J.; Latsou, D. \& Yfantopoulos, J. Oral health related quality of life of Greek adolescents: a cross-sectional study. Eur. Arch. Paediatr. Dent., 12(3):146-50, 2011.

Perea, C.; Suárez-García, M. J.; Del Río, J.; Torres-Lagares, D.; Montero, J. \& Castillo-Oyagüe, R. Oral health-related quality of life in complete denture wearers depending on their socio-demographic background, prosthetic-related factors and clinical condition. Med. Oral Patol. Oral Cir. Bucal., 18(3):e371-80, 2013.

Pereira, T. C.; Brasolotto, A. G.; Conti, P. C. \& Berretin-Felix, G. Temporomandibular disorders, voice and oral quality of life in women. J. Appl. Oral Sci., 17 Suppl.:50-6, 2009.

Reissmann, D. R.; Sierwald, I.; Heydecke, G. \& John, M. T. Interpreting one oral health impact profile point. Health Qual. Life Outcomes, 11:12, 2013.

Rosell, F. L.; Oliveira, A. L. B. M.; Tagliaferro, E. P. S.; da Silva, S. R. C. \& Valescky Júnior, A. Impacto dos problemas de saúde bucal na qualidade de vida de gestantes. Pesq. Bras. Odontoped. Clin. Integr., 13(3):287-93, 2013.

Rusanen, J.; Lahti, S.; Tolvanen, M. \& Pirttiniemi, P. Quality of life in patients with severe malocclusion before treatment. Eur. J. Orthod., 32(1):43-8, 2010.

Rusanen, J.; Silvola, A. S.; Tolvanen, M.; Pirttiniemi, P.; Lahti, S. \& Sipilä, K. Pathways between temporomandibular disorders, occlusal characteristics, facial pain, and oral health-related quality of life among patients with severe malocclusion. Eur. J. Orthod., 34(4):512-7, 2012

Sanders, A. E.; Slade, G. D.; John, M. T.; Steele, J. G.; SuominenTaipale, A. L.; Lahti, S.; Nuttall, N. M. \& Allen, P. F. A cross-national comparison of income gradients in oral health quality of life in four welfare states: application of the Korpi and Palme typology. J. Epidemiol. Community Health, 63(7):569-74, 2009a.

Sanders, A. E.; Slade, G. D.; Lim, S. \& Reisine, S. T. Impact of oral disease on quality of life in the US and Australian populations. Community Dent. Oral Epidemiol., 37(2):171-81, 2009b.

Shearer, D. M.; Thomson, W. M.; Broadbent, J. M. \& Poulton, R. Does maternal oral health predict child oral health-related quality of life in adulthood? Health Qual. Life Outcomes, 9:50, 2011.

Sischo, L. \& Broder, H. L. Oral Health-related Quality of Life. J. Dent. Res., 90(11):1264-70, 2011.

Slade, G. D. Oral health related quality of life is important for patients, but what about populations? Community Dent. Oral Epidemiol., 40(2):39-43, 2012.

Sousa, P. C.; Mendes, F. M.; Imparato, J. C. \& Ardenghi, T. M Differences in responses to the Oral Health Impact Profile (OHIP14) used as a questionnaire or in an interview. Braz. Oral Res., 23(4):358-64, 2009.

Thomson, W. M.; Caspi, A.; Poulton, R.; Moffitt, T. E. \& Broadbent, J. M. Personality and oral health. Eur. J. Oral Sci., 119(5):366-72, 2011.

Ulinski, K. G. B.; do Nascimento, M. A.; Lima, A. M. C.; Benetti, A. R.; Poli-Frederico, R. C.; Fernandes, K. B. P.; Fracasso, M. L. C. \& Maciel, S. M. Factors related to oral health-related quality of life of independent brazilian elderly. Int. J. Dent., 2013:705047, 2013.

Zanatta, F. B.; Ardenghi, T. M.; Antoniazzi, R. P.; Pinto, T. M. P. \& Rösing, C. K. Association between gingival bleeding and gingival enlargement and oral health-related quality of life (OHRQoL) of subjects under fixed orthodontic treatment: a cross-sectional study. B. M. C. Oral Health, 12:53, 2012.

Zani, S. R.; Rivaldo, E. G.; Frasca, L. C. \& Caye, L. F. Oral health impact profile and prosthetic condition in edentulous patients rehabilitated with implant-supported overdentures and fixed prostheses. J. Oral Sci., 51(4):535-43, 2009.

Zhang, Q.; Witter, D. J.; Gerritsen, A. E.; Bronkhorst, E. M. \& Creugers, $\mathrm{N}$. H. Functional dental status and oral health-related quality of life in an over 40 years old Chinese population. Clin. Oral Investig., 17(6):1471-80, 2013.

Dirección para correspondencia:

Clarisse Virginia Díaz Reissner

Odontóloga

Programa de Doctorado en Metodología de la Investigación en Ciencias Biomédicas y Salud Pública

Universitat Autònoma Barcelona

ESPAÑA

Email: diazclarisse@gmail.com

Recibido: 14-07-2016

Aceptado: 23-12-2016 